\title{
Decoding options and accuracy of translation of developmentally regulated UUA codon in Streptomyces: bioinformatic analysis
}

\author{
Ihor Rokytskyy ${ }^{\dagger}$, Oksana Koshla $^{\dagger}$, Victor Fedorenko and Bohdan Ostash ${ }^{*}$ (1)
}

\author{
*Correspondence: \\ b.ostash@Inu.edu.ua \\ †'Ihor Rokytskyy and Oksana \\ Koshla have contributed \\ equally to this work \\ Department of Genetics \\ and Biotechnology, Ivan \\ Franko National University \\ of Lviv, Hrushevskoho st. 4, \\ Lviv 79005, Ukraine
}

\begin{abstract}
Background: The gene bldA for leucyl tRNA ${ }^{\text {Leu }}$ UAA is known for almost 30 years as a key regulator of morphogenesis and secondary metabolism in genus Streptomyces. Codon UUA is the rarest one in Streptomyces genomes and is present exclusively in genes with auxiliary functions. Delayed accumulation of translation-competent tRNA ${ }^{\text {Leu }}$ UAA is believed to confine the expression of UUA-containing transcripts to stationary phase. Implicit to the regulatory function of UUA codon is the assumption about high accuracy of its translation, e.g. the latter should not occur in the absence of cognate tRNA ${ }^{\text {Leu }}$ UAA. However, a growing body of facts points to the possibility of mistranslation of UUA-containing transcripts in the bldA-deficient mutants. It is not known what type of near-cognate tRNA(s) may decode UUA in the absence of cognate tRNA in Streptomyces, and whether UUA possesses certain inherent properties (such as increased/decreased accuracy of decoding) that would favor its use for regulatory purposes.
\end{abstract}

Findings: Here we took bioinformatic approach to address these questions. We catalogued the entire complement of tRNA genes from several relevant Streptomyces and identified genes for posttranscriptional modifications of tRNA that might be involved in UUA decoding by cognate and near-cognate tRNAs.

Conclusions: Based on tRNA gene content in Streptomyces genomes, we propose possible scenarios of UUA codon mistranslation. UUA is not associated with an increased rate of missense errors as compared to other leucyl codons, contrasting general belief that low-abundant codons are more error-prone than the high-abundant ones.

Keywords: Streptomyces, bldA, tRNA, UUA codon, Codons, Models of translation

\section{Findings}

The bldA mutants of Streptomyces coelicolor A3(2) were first isolated almost 40 years ago (Merrick 1976) and 11 years later were shown to carry mutations within gene for leucyl tRNA ${ }_{\text {UAA. }}^{\text {Le }}$ (Lawlor et al. 1987) This mutation abolishes aerial mycelium formation (Bald phenotype) and antibiotic production by streptomycetes; currently bldA is extensively used as a tool to activate cryptic secondary metabolome (Hackl and Bechthold

(c) 2016 The Author(s). This article is distributed under the terms of the Creative Commons Attribution 4.0 International License (http://creativecommons.org/licenses/by/4.0/), which permits unrestricted use, distribution, and reproduction in any medium, provided you give appropriate credit to the original author(s) and the source, provide a link to the Creative Commons license, and indicate if changes were made. 
2015). Codon TTA, whose decoding is controlled by bldA, is very rare in GC-rich Streptomyces genomes, and present only in genes with unknown and auxiliary functions, such as colony morphological development and antibiotic production. As accumulation of translation-competent, charged tRNA ${ }^{\text {Leu }}{ }_{\text {AA }}$ is confined to late stages of growth, so does the expression of TTA-containing genes (Chater 2006). UUA codon and its cognate tRNA were long time ago suggested to form a genetic switch that operates at the level of translation (Hopwood 1987). Use of TTA for regulatory purposes is somewhat controversial. On one hand, rarity of this codon ensures that only certain genes are influenced by bldA-based switch. On the other hand, rare codons are thought to be associated with higher missense error rates, which would not favor their proper operation as a switch. Although it was suggested implicitly that UUA is decoded accurately, there is a number of notable exceptions. Particularly, bldA mutants showed no Bald phenotype on certain solid media (Hopwood 1987); several TTA-containing genes were expressed in bldAdeficient strains (Trepanier et al. 2002; Makitrynskyy et al. 2013), particularly when their transcription is artificially elevated (Gramajo et al. 1993). All these observations imply that efficient mistranslation of UUA codon is possible at least under some conditions. It is not known what tRNAs could potentially recognize UUA in the absence of tRNA ${ }^{\text {Leu }}$ UAA and what structural and functional peculiarities of tRNA ${ }_{\text {UAA }}^{\text {Leu }}$ contribute to its regulatory function (Pettersson and Kirsebom 2011). Here we took bioinformatic approach to obtain new insight into this issue and to chart new directions for experimental verification.

Although there are several databases of tRNA genes, such as GtRNAdb and tRNADBCE, they provide contradictory information on tRNA content for model strain Streptomyces coelicolor A3(2) and lack data on several species relevant to this work. Furthermore, available online resources do not show what kind of tRNA may decode certain codon via wobble interaction. We therefore compiled all available information on tRNA genes and their decoding capacity for six Streptomyces species with known cases of bldA-based regulation using several databases and search tools detailed in Additional file 1. It could be concluded that overall tRNA gene content is highly conserved in six analyzed Streptomyces genomes, although copy number of individual tRNA genes varies (Table 1). For several codons there were no acceptor tRNAs (for example, alanine codon GCT); those apparently are recognized by isoacceptor tRNAs (e.g. GCT is read by GCC isoacceptor; see Table 1), which are encoded within the analyzed genomes. It is common for all known organisms that the entire set of sense codons $(61+1$ initiator) is read by far fewer than 62 isoacceptors; extreme cases of anticodon-sparing are documented in some archaea and mycoplasmas, where only 26-33 anticodons are required to read the genetic code (Marck and Grosjean 2002). All genomes contain single tRNA gene for UUA decoding. Therefore, differences in bldA mutant phenotypes across different species could not be ascribed to variations in tRNA gene content. Codon UUA could be poorly recognized by phenylalanine tRNA ${ }^{\text {Phe }}$ AAA via wobble interactions (Lim and Curran 2001). However, no respective tRNA gene is present in all studied Streptomyces genomes. Cytidine posttranscriptionally modified with lysidine $\left(\mathrm{k}^{2} \mathrm{C}_{34}\right)$ is known to recognize adenosine in third codon position. This kind of modification to date was described only for anticodon CAU, which normally decodes methionine codon AUG. The $\mathrm{k}^{2} \mathrm{C}_{34}$-containing tRNA ${ }^{\mathrm{CAU}}$ is charged with isoleucine and recognizes isoleucine 


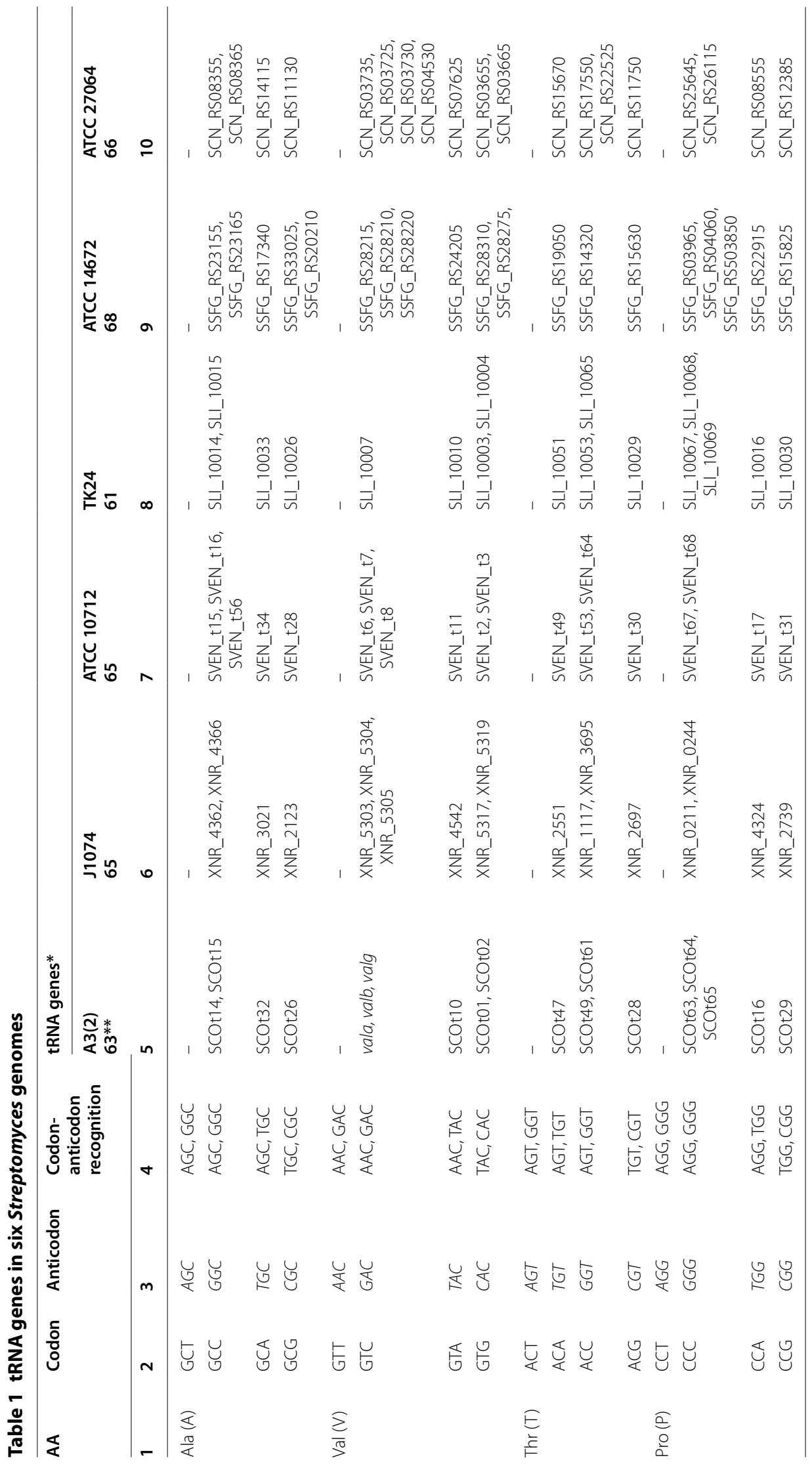




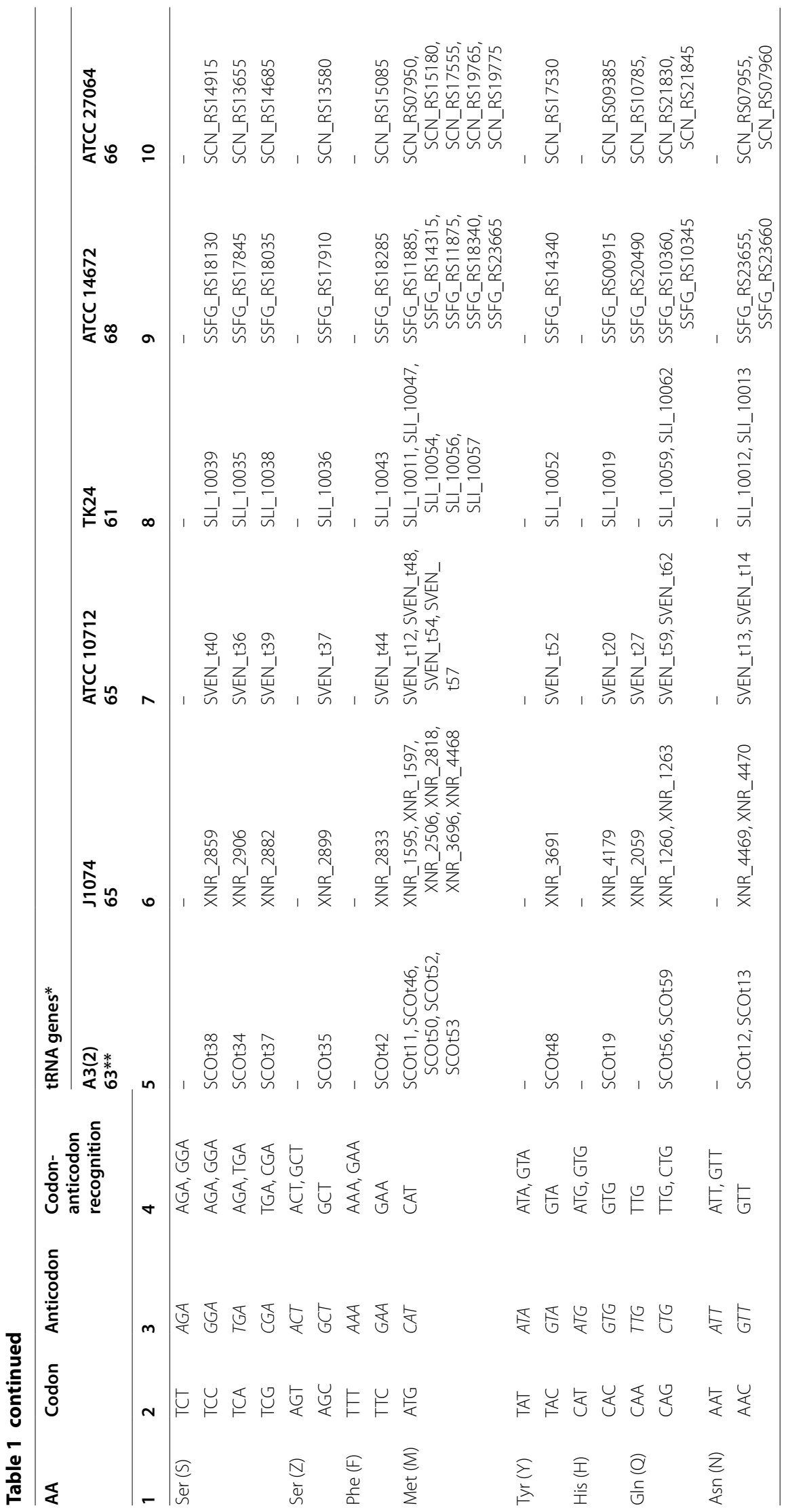




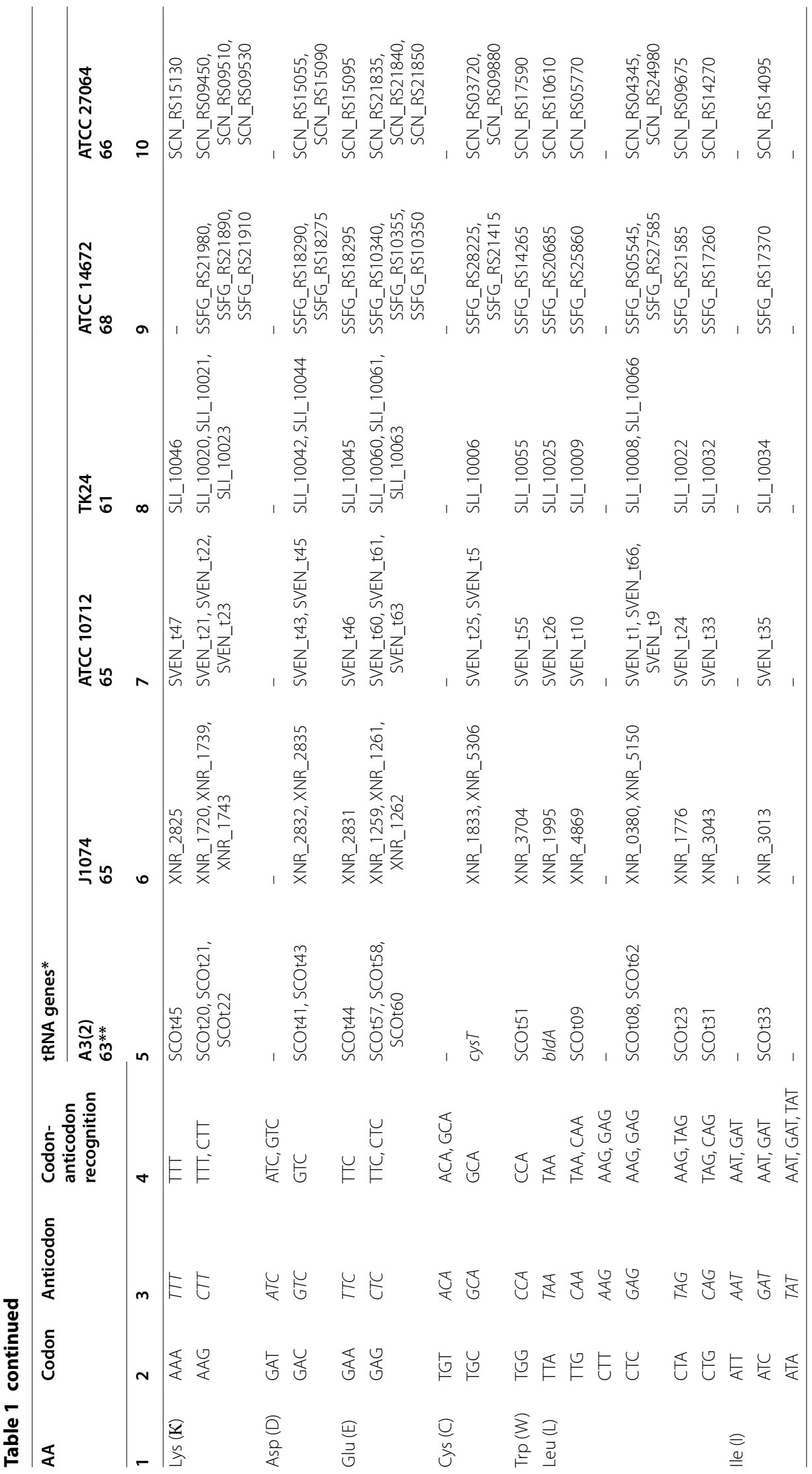




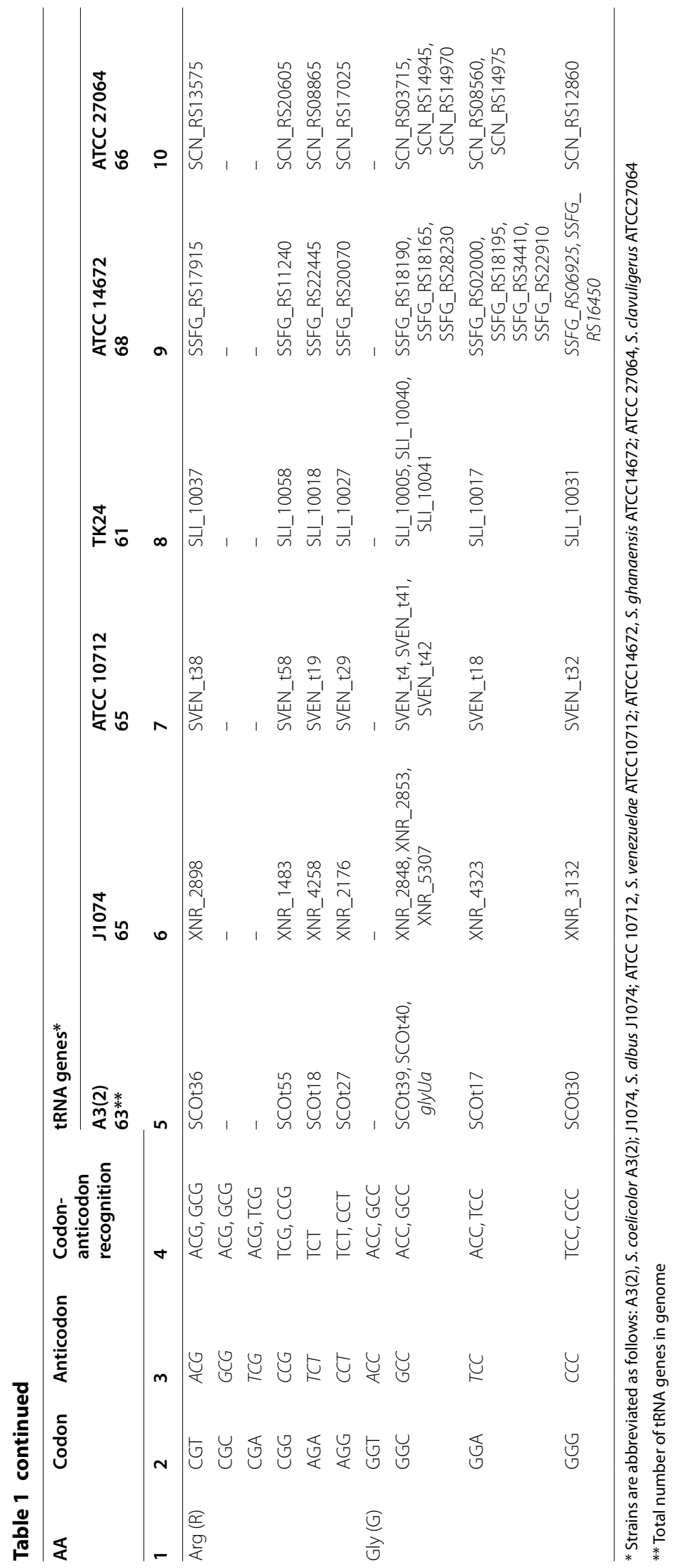


codon AUA. It is not possible for tRNA ${ }^{\mathrm{k} 2 \mathrm{CAU}}$ Ile to recognize codon UUA because of mismatch in second codon position. Hence, there are no tRNA genes in Streptomyces genomes that would allow UUA codon reading (via correct or wobble interactions) in the absence of cognate tRNA ${ }^{\mathrm{UAA}}{ }_{\text {Leu. }}$. We therefore looked into possibility of UUA misreading. According to Lim and Curran (2001), three anticodons could misread UUA: UAC, GAA, CAA. Of these, first two would lead to aminoacid misincorporation (Val and Phe, respectively).

To gain initial insight into relative mistranslation rate associated with bldA, we applied a computational model of translation accuracy (Shah and Gilchrist 2010) that deduces ratio of abundances of cognate to near-cognate tRNAs (differ from cognate one by one mismatch; see Table 2). The rationale is that error rate would depend not only on the abundance of cognate tRNA, but also on the abundance of all near-cognates, that

Table 2 Gene copy number (GCN) for tRNA genes in six Streptomyces genomes

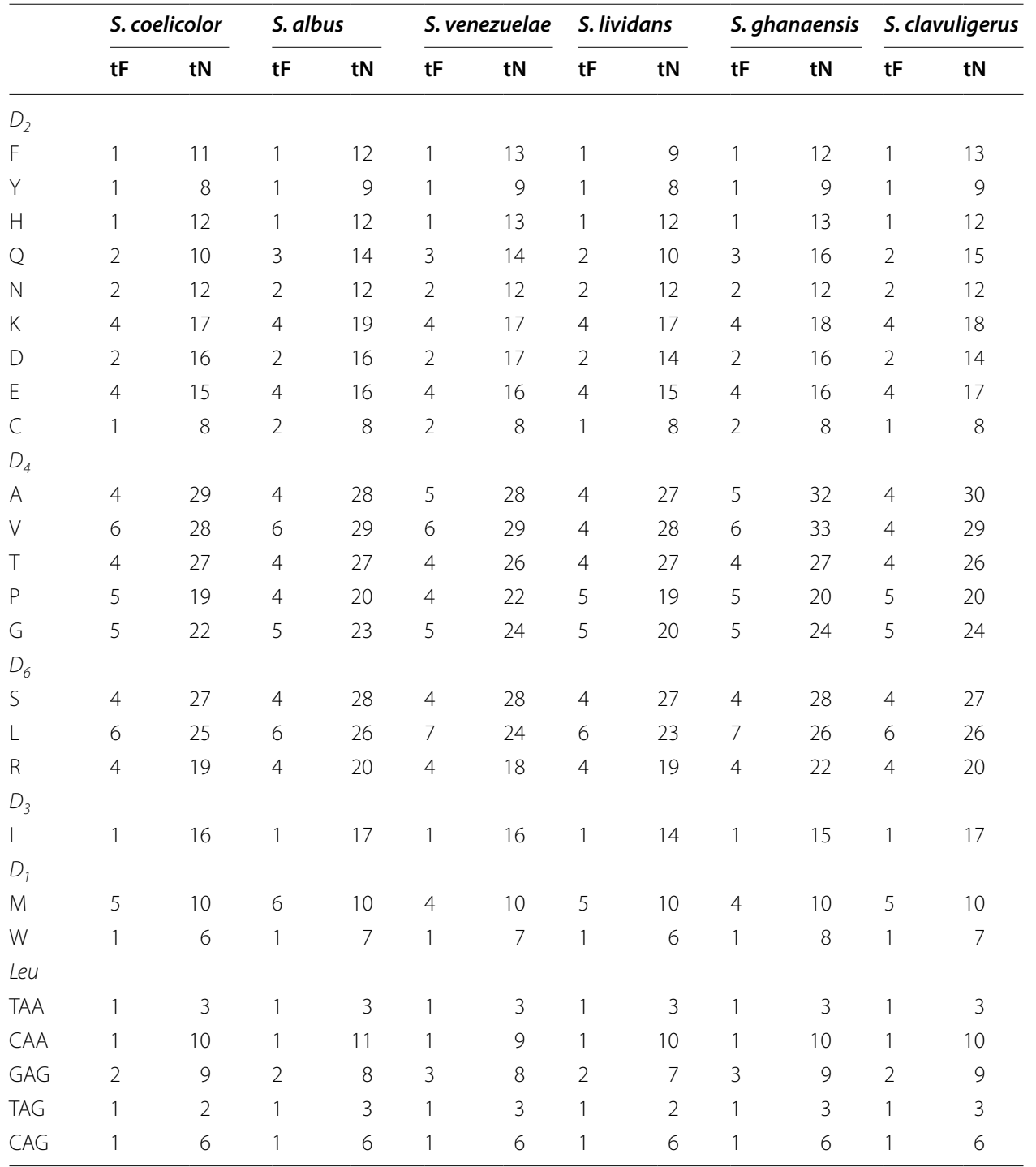


compete with the former for codon recognition. There was statistically significant positive correlation between the abundance of all leucyl tRNAs and their near-cognates for six Streptomyces species (Fig. 1), suggesting that error rates should not differ for different Leu codon-cognate tRNA pairs (if so, abundances of cognates and near-cognates would be uncorrelated). Similar correlation pattern was observed for most Streptomyces tRNAs (Additional file 1: Fig. S1, S2). We further calculated elongation and error rates for all six leucine codons and revealed that UUA had, in fact, the lowest missense error rate (Table 3). Our findings contrast general belief that low-abundant tRNAs are associated with higher mistranslation rates. Yet, they extend the nuanced view of codon accuracy, based originally on non-actinobacterial, low-GC (less than $70 \%$ ) genomes (Shah and Gilchrist 2010), onto GC-rich streptomycetes. Our data also agree with the expectation that proper operation of codon-based genetic switch should be based on accurate translation of UUA.

Rather narrow options for UUA mistranslation, revealed by our analysis, did not take into account that decoding properties of tRNAs can be tuned via posttranscriptional modifications. We identified in genomes of two model streptomycetes a large set of genes for such modifications (including $\mathrm{k}^{2} \mathrm{C}$; see above), seven of which are involved in maturation of nascent tRNA ${ }^{\mathrm{UAA}}{ }_{\text {Leu }}$ in various non-actinomycete bacteria (Table 4 and Additional file 1: Fig. S3). Of particular interest are the genes for modification of anticodon loop and adjacent bases of tRNA ${ }^{\mathrm{UAA}}$ Leu (see Additional file 1). For example, it is possible that a posttranscriptional modification of nascent bldA transcript important for UUA decoding and/or tRNA ${ }_{\text {Leu }}$ maturation is delayed in Streptomyces. It would temporally limit the occurrence of translationally-competent tRNA ${ }_{\text {Lew }}^{\mathrm{UAA}}$ thus explaining late expression of TTA-containing genes. If so, then streptomycetes deficient in certain tRNA modification genes would resemble bld mutants. We are currently studying this idea using $S$. albus and S. ghanaensis as experimental models and invite verification of this conjecture for other strains. As a conclusion, our work shows that there are no theoretical grounds to consider UUA more error prone than the other leucine codons. We examined, in silico, options for UUA mistranslation and draw the attention of researchers to poorly understood aspects of function of bldA genetic switch.

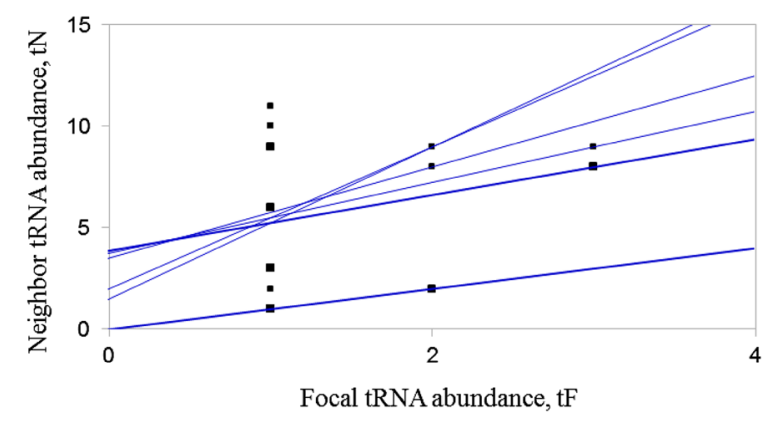

Fig. 1 Correlation between a focal leucyl tRNA's abundance tF and the abundance of its neighbors tN, across six Streptomyces genomes (see Additional file 1 for details). Each point represents a leucine tRNA species. The solid lines represent the regression lines between $\mathrm{t} F$ and $\mathrm{tN}$ for each genome. The data are dependent and nonrandom (Wilcox test, 0.042), and positively correlated (Spearman coefficient, min. 0.354). The mean of the distribution of correlation coefficient values for leucine codons differ from 0 (see Additional file 1: Fig. S2) 


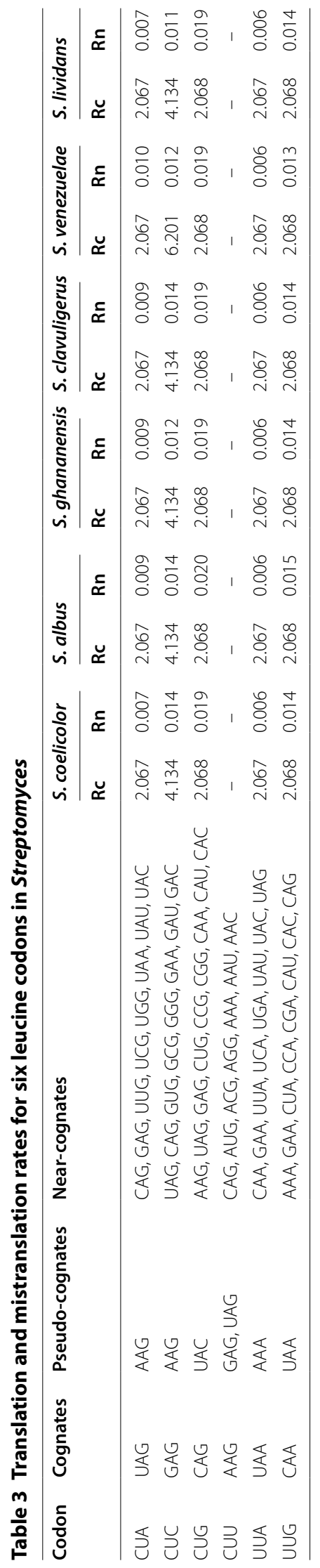


Table 4 Genes for tRNA posttranscriptional modification in S. coelicolor and S. albus genomes

\begin{tabular}{|c|c|c|c|}
\hline Protein & $\begin{array}{l}\text { S. coelicolor } \mathrm{A} 3(2) \\
\text { homolog }\end{array}$ & $\begin{array}{l}\text { S. albus J1074 } \\
\text { homolog }\end{array}$ & Annotation \\
\hline IscS & SCO5486 & XNR_1347 & tRNAsulfurtransferase, PLP-dependent \\
\hline IscU & SCO1920 & XNR_4942 & iron-sulfur cluster assembly enzyme \\
\hline TruA & SCO4731 & XNR_3758 & tRNApseudouridine(38-40) synthase \\
\hline TruB & SCO5709 & XNR_1143 & tRNApseudouridine synthase B \\
\hline TruC & SCO1625 & XNR_4806 & tRNApseudouridine synthase C \\
\hline DusB & SCO2497 & XNR_4421 & tRNA-dihydrouridine synthase B \\
\hline $\operatorname{TrmA}$ & SCO5901 & XNR_0992 & tRNAm5(U34)methyltransferase \\
\hline $\operatorname{TrmB}$ & SCO4111 & XNR_2813 & tRNA (guanine-N(7)-)-methyltransferase \\
\hline $\operatorname{TrmD}$ & SCO5594 & XNR_1214 & tRNA m(1)G37 methyltransferase, SAM-dependent \\
\hline $\operatorname{TrmH}$ & SCO4236 & XNR_2558 & tRNA mG18-2'-O-methyltransferase, SAM-dependent \\
\hline RluA & SCO2073 & XNR_4806 & $\begin{array}{l}\text { 23S rRNApseudouridine(746), tRNApseudouridine(32) } \\
\text { synthase, SAM-dependent }\end{array}$ \\
\hline TadA & SCO4038 & XNR_2881 & tRNA-specific adenosine deaminase \\
\hline FolE & SCO3403 & XNR_3431 & GTP cyclohydrolase I \\
\hline QueA & SCO1804 & XNR_5018 & S-adenosylmethionine:tRNAribosyltransferase-isomerase \\
\hline MnmA & SCO5488 & XNR_1345 & tRNA(GIn,Lys,Glu) U34 2-thiouridylase \\
\hline MiaA & SCO5791 & XNR_1074 & $\begin{array}{l}\text { delta(2)-isopentenylpyrophosphatetRNA-adenosine } \\
\text { transferase }\end{array}$ \\
\hline MiaB & SCO5787 & XNR_1078 & tRNA-i(6)A37 methylthiotransferase \\
\hline AroA & SCO6819 & XNR_1588 & 5-Enolpyruvylshikimate-3-phosphate synthetase \\
\hline AroB & SCO1494 & XNR_5357 & 3-Dehydroquinate synthase \\
\hline AroC & SCO1496 & XNR_5355 & Chorismate synthase \\
\hline AroE & SCO1498 & XNR_5354 & Dehydroshikimatereductase, NAD(P)-binding \\
\hline AroD & SCO1961 & XNR_4909 & 3-Dehydroquinate dehydratase \\
\hline AroK & SCO1495 & XNR_5356 & Shikimate kinase I \\
\hline TsaA & SCO5032 & XNR_4120 & $\begin{array}{l}\text { tRNA-Thr(GGU) m(6)t(6)A37 methyltransferase, SAM- } \\
\text { dependent }\end{array}$ \\
\hline TsaB & SCO4750 & XNR_3789 & $\begin{array}{l}\text { tRNA(ANN) t(6)A37 threonylcarbamoyladenosine modifi- } \\
\text { cation protein; binding partner and protease for TsaD }\end{array}$ \\
\hline $\mathrm{TsaC}$ & SCO5362 & XNR_1471 & $\begin{array}{l}\text { t(6)A37 threonylcarbamoyladenosine biosynthesis } \\
\text { protein }\end{array}$ \\
\hline TsaD & SCO4752 & XNR_3791 & $\begin{array}{l}\text { tRNA(ANN) t(6)A37 threonylcarbamoyladenosine modifi- } \\
\text { cation protein; glycation binding protein }\end{array}$ \\
\hline TsaE & SCO4747 & XNR_3786 & $\begin{array}{l}\text { tRNA(ANN) t(6)A37 threonylcarbamoyladenosine modifi- } \\
\text { cation protein; ADP binding protein }\end{array}$ \\
\hline Tils & SCO3406 & XNR_3428 & tRNA(Ile)-lysidinesynthetase \\
\hline
\end{tabular}

\section{Additional file}

Additional file 1. Codon correlations and proposed Leu-tRNA modifications.

\section{Authors' contributions}

IR generated tN/tF data and carried out computational analysis of codon accuracy; OK analyzed tRNA and tRNA modifcation genes in Streptomyces genomes; VF and $\mathrm{BO}$ analyzed data; IR and $\mathrm{BO}$ drafted the manuscript; all authors read and approved the final manuscript. 


\section{Competing interests}

The authors declare that they have no competing interests.

Received: 31 December 2015 Accepted: 28 June 2016

Published online: 04 July 2016

\section{References}

Chater KF (2006) Streptomyces inside-out: a new perspective on the bacteria that provide us with antibiotics. Philos Trans R Soc Lond B Biol Sci 361:761-768. doi:10.1098/rstb.2005.1758

Gramajo H, Takano E, Bibb M (1993) Stationary-phase production of the antibiotic actinorhodin in Streptomyces coelicolor A3(2) is transcriptionally regulated. Mol Microbiol 7(6):837-845. doi:10.1111/j.1365-2958.1993.tb01174. X

Hackl S, Bechthold A (2015) The gene bldA, a regulator of morphological differentiation and antibiotic production in Streptomyces. Arch Pharm 348:455-462. doi:10.1002/ardp.201500073

Hopwood DA (1987) The Leeuwenhoek lecture. Towards an understanding of gene switching in Streptomyces, the basis of sporulation and antibiotic production. Proc R Soc Lond B Biol Sci 235:121-138. doi:10.1098/rspb.1988.0067

Lawlor E, Baylis H, Chater K (1987) Pleiotropic morphological and antibiotic deficiencies result from mutations in a gene encoding a tRNA-like product in Streptomyces coelicolor A3(2). Genes Dev 1:1305-1310. doi:10.1101/gad.1.10.1305

Lim VI, Curran JF (2001) Analysis of codon: anticodon interactions within the ribosome provides new insights into codon reading and the genetic code structure. RNA 7:942-957

Makitrynskyy R, Ostash B, Tsypik O, Rebets Y, Doud E, Meredith T, Luzhetskyy A, Bechthold A, Walker S, Fedorenko V (2013) Pleiotropic regulatory genes bldA, adpA and $a b s B$ are implicated in production of phosphoglycolipid antibiotic moenomycin. Open Biol 3:130121. doi:10.1098/rsob.130121

Marck C, Grosjean H (2002) tRNomics: analysis of tRNA genes from 50 genomes of Eukarya, Archaea, and Bacteria reveals anticodon-sparing strategies and domain-specific features. RNA 8:1 189-1232. doi:10.1017/S1355838202022021

Merrick M (1976) A morphological and genetic mapping study of bald colony mutants of Streptomyces coelicolor. J Gen Microbiol 96:299-315. doi:10.1099/00221287-96-2-299

Pettersson BM, Kirsebom LA (2011) tRNA accumulation and suppression of the bldA phenotype during development in Streptomyces coelicolor. Mol Microbiol 79:1602-1614. doi:10.1111/j.1365-2958.2011.07543.x

Shah P, Gilchrist MA (2010) Effect of correlated tRNA abundances on translation errors and evolution of codon usage bias. PLoS Genet 6:e1001128. doi:10.1371/journal.pgen.1001128

Trepanier N, Jensen S, Alexander D, Leskiw B (2002) The positive activator of cephamycin C and clavulanic acid production in Streptomyces clavuligerus is mistranslated in a bldA mutant. Microbiology 148:643-656. doi:10.1099/00221287-148-3-643

\section{Submit your manuscript to a SpringerOpen ${ }^{\circ}$ journal and benefit from:}

- Convenient online submission

Rigorous peer review

- Immediate publication on acceptance

- Open access: articles freely available online

- High visibility within the field

- Retaining the copyright to your article

Submit your next manuscript at $\boldsymbol{\nabla}$ springeropen.com 